\title{
The study of the effect of energy dissipation at fatigue crack tip on its propagation rate
}

\author{
by A. Iziumova*, A. Vshivkov*, O. Plekhov*
}

* Institute of continuous media mechanics UB RAS, 1 Ac. Koroleva Str., Perm, Russia, poa@icmm.ru

\begin{abstract}
This paper is devoted to application of infrared thermography to study heat dissipation at crack tip with a goal to derive an energy based fatigue crack propagation law. During experimental work, metallic specimens with three types of geometries were investigated. To verify the results of heat souses calculation carried out based on infrared thermography data the original heat flux gauge was used. According to the obtained data two different stages of crack propagation could be distinguished for all studied samples. Based on it we derived two relations between crack rate and heat dissipation rate for both stages.
\end{abstract}

\section{Introduction}

Nowadays it is well known that the process of fatigue crack propagation in metals is accompanied by the formation of the areas of monotonic and cyclic plastic deformation. The intense strain localization in these areas leads to the noticeable temperature perturbation at crack tip. The infrared thermography (IRT) is used more than thirty years for estimation of linear fracture mechanics parameters (for instance, stress intensity factor (SIF)) and forecast the crack rate based on value of SIF (Paris's law). But from physical point of view the application of the SIF to describe the fatigue crack rate in metals requests the small scale yielding assumption. The possibilities of IRT are significantly wilder than thermal stress analysis and allow one to study the heat dissipation caused by plastic deformation.

Many authors [1-3] have proposed alternative approach to determine the crack rate as a function of J-integral or plastic deformation zone size or the value of dissipated energy, etc. The use of the energy approach in the description of fatigue crack propagation originates in [4-11]. One of the most detailed descriptions of energy balance at the fatigue crack tip was proposed in [11]. A similar relation was obtained in [12] where the law of crack propagation was derived as entropy production in the system written as product of driving force responsible for the translational mode on the corresponding thermodynamic flow. In [13], the authors experimentally demonstrated the relationship between the work of plastic deformation and the change in the stress intensity factor. Based on the assumption that most part of this work is converted into heat they derived the fatigue crack propagation law.

Deriving of relations based on analysis of energy balance ahead of a crack tip requires the development of simple technique to obtain data of heat dissipation rate (or, in the best case, stored energy rate) and crack growth rate directly from fatigue test. It could allow one to classify the stages of crack propagation and to monitor the state of materials on the base of energy approach. Infrared thermography can be considered as such technique but It application requests a data treatment with goal to estimate the power of heat dissipation. In this work the original contact heat flux gauge was developed to detect energy dissipation value during crack propagation and verify the data of infrared thermography. This gauge is based on the Seebeck effect and includes two Pelietier elements and temperature controlling feedback. This gauge allows us to study in details a dissipated energy evolution in metal samples (AISI304 and titanium alloy Ti-0.8AI$0.8 \mathrm{Mn}$ ) with three different geometries and support propose a relations between heat dissipation and fatigue crack rate.

\section{Materials and experimental conditions}

Experimental study of heat dissipation was carried out on the plane specimens of titanium alloy Ti-0.8Al-0.8Mn and stainless steel AISI304. The specimen geometries are presented in Figure 1 (type a - titanium alloy specimens; type $\mathrm{b}$ and $\mathrm{c}$ - stainless steel specimens). All sizes are presented in millimeters.

CT (Compact Tension) specimens (Figure 1a) were studied under fatigue conditions with stress ration $\mathrm{R}=0.1$ and loading frequency $10 \mathrm{~Hz}$. Experiments were carried out on servo-hydraulic testing machine Instron 8802 under constant loading amplitude of 2.5 and $3 \mathrm{kN}$. Crack length was measured by crack opening displacement (COD) gauge 2670-116. These experiments were carried out in collaboration with Laboratory of physics of strength of materials and intelligent diagnostic systems, Togliatti State University (Togliatti, Russia).

Fatigue test also was performed on SEN (Single Edge Notched) specimens (Figure 1b) under constant loading amplitude of 8.5 and $8.8 \mathrm{kN}$. Experiments were carried out on servo-hydraulic testing machine with a DOLI EDC 580 controller at a frequency of $20 \mathrm{~Hz}$ and stress ratio $\mathrm{R}=0$. Crack length was measured by potential drop method (PDM). Experiments on this type of specimens were carried out in collaboration with University of the Federal Armed Forces Munich (Neubiberg, Germany). 


\subsection{1/qirt.2016.101}

Specimens of dog bone shape with a central hole (Figure 1c) were studied under cyclic loading conditions $(R=-0.03)$ with loading amplitude decreasing from 17 to $13 \mathrm{kN}$ with step of $2 \mathrm{kN}$. Cyclic loading was stopped when heat flux started to growth then loading amplitude was decreased and experiment was continued. Loading frequency was $10 \mathrm{~Hz}$. PDM was used to measure crack length during mechanical test. Mechanical tests were carried out using a $100 \mathrm{kN}$ servohydraulic machine Bi-00-100.

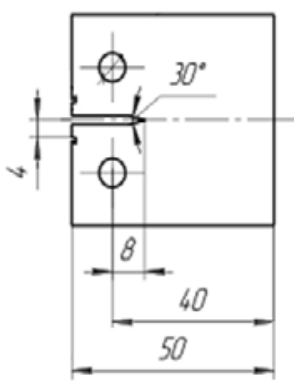

a)

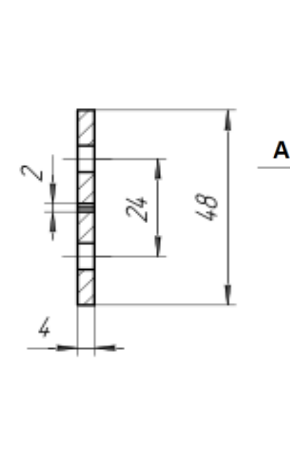

Fig. 1. Geometries of studied specimens: a) titanium alloy specimens; b) and c) stainless steel specimens

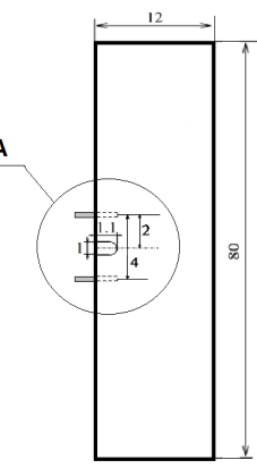

b)
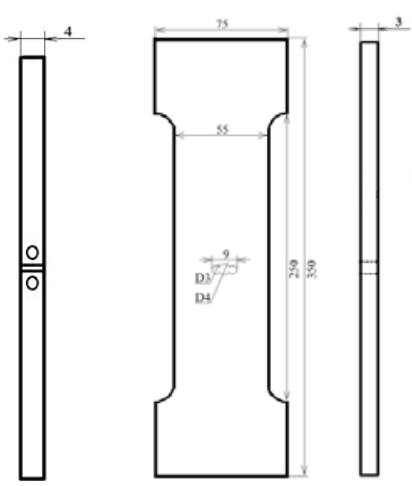

c)

The surfaces of the specimens were polished in several stages by the abrasive paper (at the final stage of polishing the grit size does not exceed $3 \mu \mathrm{m}$ ). Before starting the experiment the polished surface was covered by a thin layer of amorphous carbon. The temperature evolution was recorded with frequency in ten times higher than loading frequency. The spectral range was 3-5 $\mu \mathrm{m}$, the spatial resolution is $10^{-4}$ meters. Calibration of the camera was made based on the standard calibration table. It was used FLIR SC5000 MW G1 F/3.0 close-up lens (distortion is less than 0.5\%) to investigate the plastic zone in details.

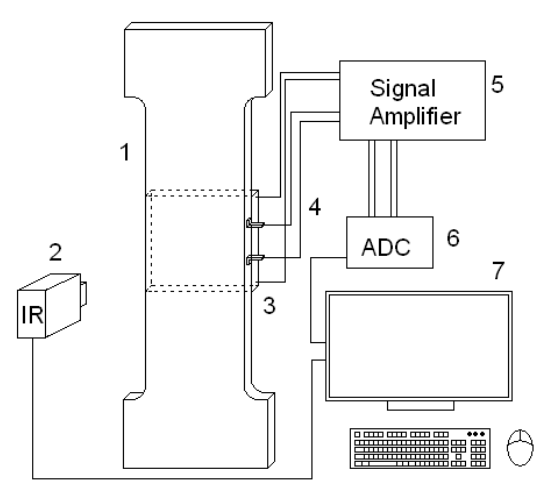

Fig. 2 Principal scheme of experimental setup.

The feature of this work is additional using the original contact gauge of heat flux which is described in details in [14]. This gauge consists of two Peltier elements. One of them plays a role of heat stabilizer. Another one is used for measuring of potential drop $U$ (in Volts) which appears because of the temperature difference between plates of Peltier element. Dependence between potential drop in Volts and heat flux in Watts is linear and it is determined by calibration function which is defined from calibration experiments with heat flux gauge. Contact gauges of heat flux with specific sizes were manufactured for each type of geometries. To provide a good thermal contact the specimen surface under heat flux gauge was coated by thermal paste.

\section{Experimental results}

At the beginning of data processing procedure, the first frame was subtracted from the film to eliminate the influence of infrared radiation from the camera lens on the determined temperature field. Due to the relative motion of the specimen with crack and infrared camera lens under cyclic tests, there is the problem of motion compensation in order to obtain the correct temperature data at a given point on specimen surface. Compensation of relative motion was made based on the algorithm described in details in [15]. The main idea of this algorithm is the selection of marker-zone on the studied surface and searching this area on the surface in each next time step. Farther, displacement of each point on the surface is calculated for each time step. As a result of data processing, we obtained the temperature increment field (Figure 3) based on which the heat sources field was determined. 


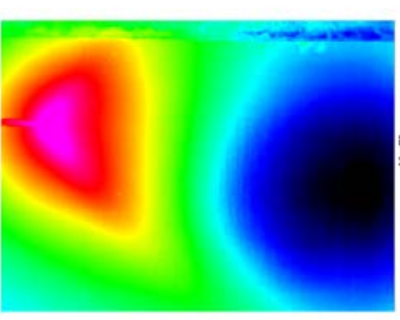

a)

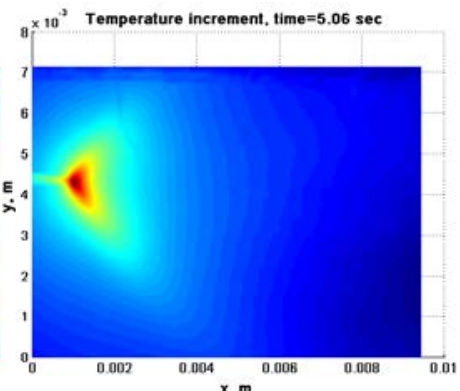

b)

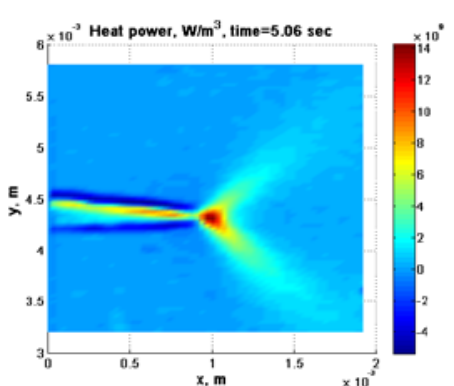

c)

Fig. 3. a) Infrared image of the sample with crack before data processing and b) obtained temperature increment field, c) heat power field near the crack tip.

To calculate the specific power of the heat source, we have used a finite difference scheme of the equation for heat sources evolution

$$
s=\rho c\left(\dot{T}+\frac{T}{\tau}\right)-k \Delta T,
$$

where $\mathrm{T}$ - temperature, $\rho$ - density, $\mathrm{c}$ - heat capacity, $\mathrm{k}$ - heat conductivity, $\mathrm{s}-$ unknown specific power of the heat source, $\tau$ - constant related to the losses of heat by heat exchange with the surroundings.

Figure $3 c$ presents heat distribution at crack tip. The calculation of heat distribution from infrared data requests a lot computing time and it is not applicable for long fatigue test. As a result we used the heat source calculation for control of contact sensor data and record the energy dissipation evolution based on contact heat flux sensor.

Figures 4-6 show data typically obtained in experiment for all types of investigated geometries and metals: heat flux and crack length versus time and crack growth rate versus range of stress intensity factor (SIF).

Despite of different testing conditions and different studied materials obtained data of heat flux show approximately the same evolution. So that, heat dissipation process associated with crack growth could be divided into two parts (stages) which are indicated by blue and black colors in Figures 3-5. The first one corresponds to the approximately constant value of heat flux and small changes of crack length. The second one is characterized of avalanche growth of heat flux and significant changes in crack length.

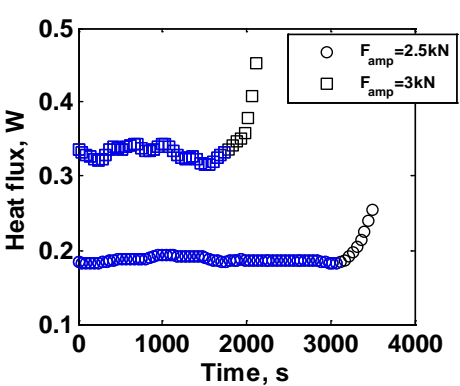

a)

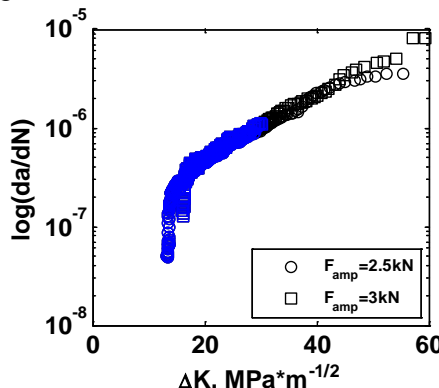

b)

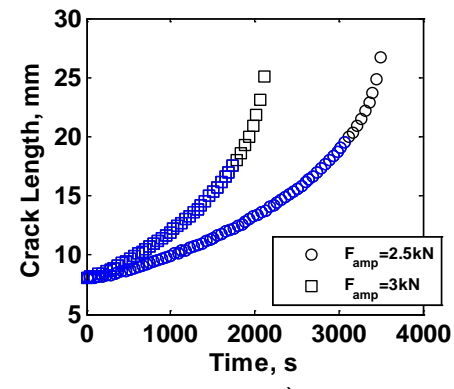

c)

Fig. 4. Experimental data for CT specimens of titanium alloy Ti-0.8Al-0.8Mn (Figure 1a): a) heat flux during fatigue test; b) crack growth rate versus range of SIF; c) crack length during fatigue test

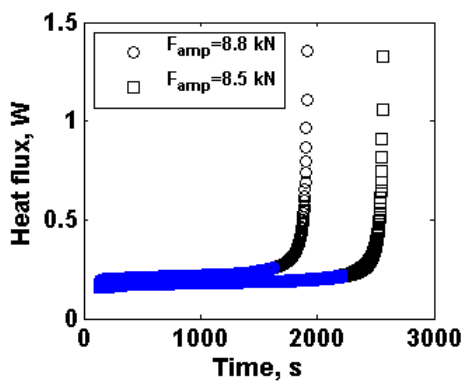

a)

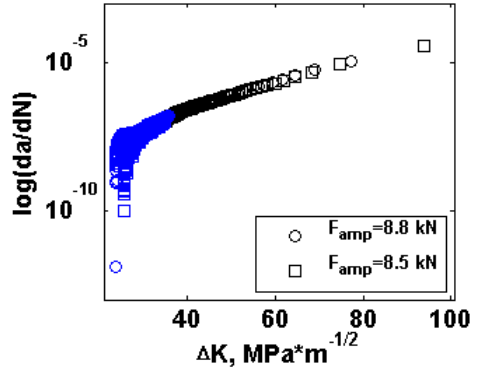

b)

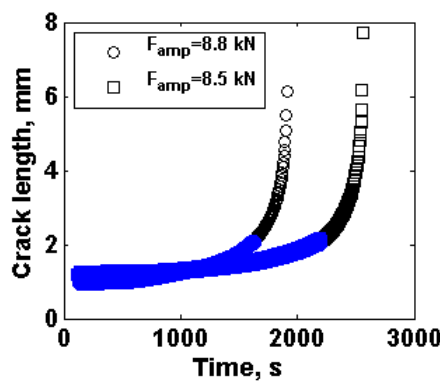

c)

Fig. 5. Experimental data for SEN specimens of stainless steel AISI304 (Figure 1b): a) heat flux during fatigue test; $b$ ) crack growth rate versus range of SIF; c) crack length during fatigue test 


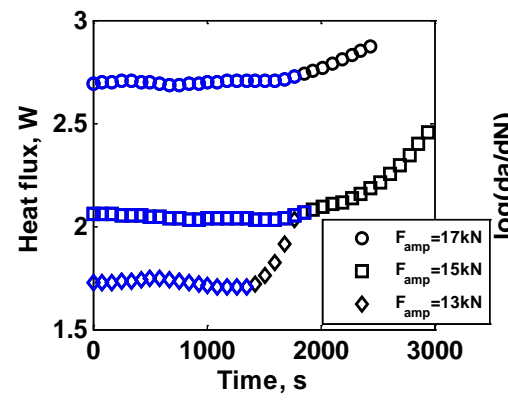

a)

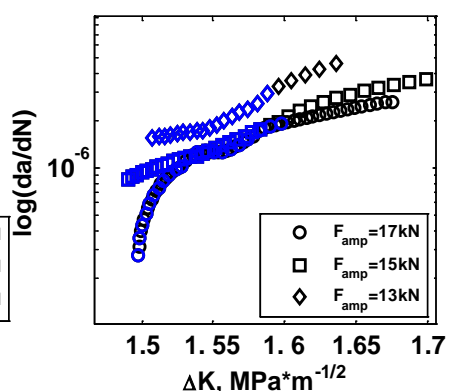

b)

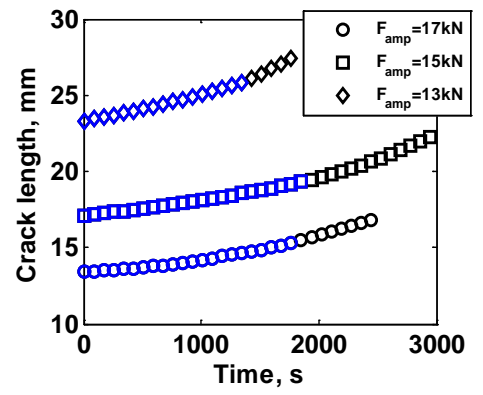

C)

Fig. 6. Experimental data for specimens of dog bone shape with a central hole of stainless steel AISI304 (Figure 1c): a) heat flux during fatigue test; b) crack growth rate versus range of SIF; c) crack length during fatigue test

It should be noted that significant changes in heat flux begins approximately on middle of linear part of Paris curve. We can assume that irreversible changes in materials with fatigue crack are started earlier than it can be observed using Paris law, but these changes are reflected on heat dissipation in crack tip area what can be detected by heat flux gauge.

\section{Processing of experimental data}

To compare two stages of crack propagation about which was said above, experimental data were normalized as it is shown in expression (1).

$$
Q_{1} a_{1}^{\prime}=\frac{Q_{1} a_{1}-Q_{1} a_{1}^{\min }}{Q_{1} a_{1}^{\text {max }}-Q_{1} a_{1}^{\min }}, Q_{2}^{\prime}=\frac{Q_{2}-Q_{2}^{\min }}{Q_{2}^{\max }-Q_{2}^{\min }}, \Delta K_{i}^{\prime}=\frac{\Delta K_{i}-\Delta K_{i}^{\min }}{\Delta K_{i}^{\max }-\Delta K_{i}^{\text {min }}}, i=1,2
$$

where $Q$, a and $\mathrm{K}$ are heat flux, crack length and stress intensity factor, respectively. Indexes indicate stages of heat dissipation process.

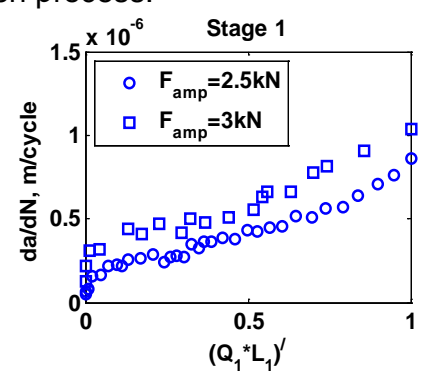

a)

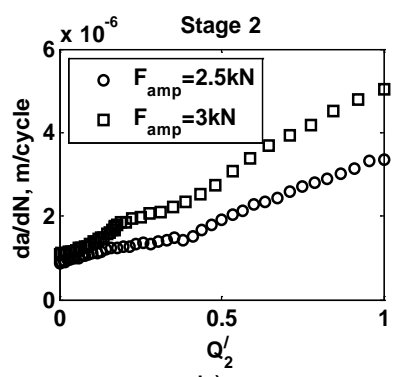

b)

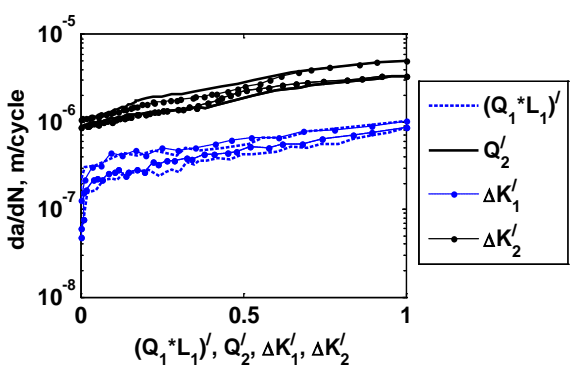

c)

Fig. 7. The dependence between crack growth rate and normalized data of heat flux multiplying by crack length on the first stage of dissipation process (a) and normalized data of heat flux on the second stage of heat dissipation (b); c comparison of normalized data with curve of fatigue crack growth. Data obtained on CT specimens

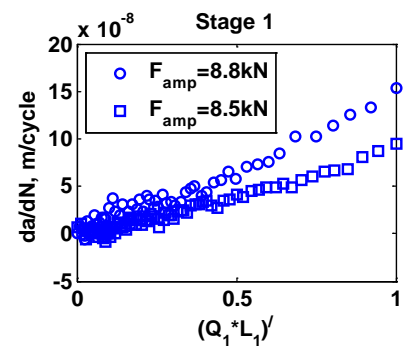

a)

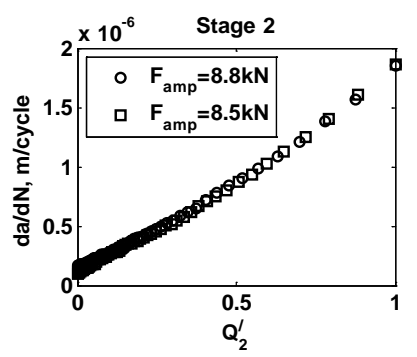

b)

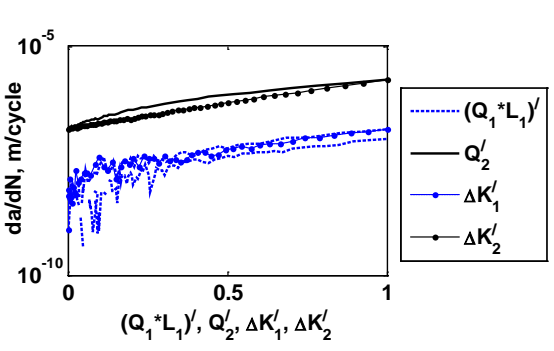

c)

Fig. 8. The dependence between crack growth rate and normalized data of heat flux multiplying by crack length on the first stage of dissipation process (a) and normalized data of heat flux on the second stage of heat dissipation (b); ccomparison of normalized data with curve of fatigue crack growth. Data obtained on SEN specimens 


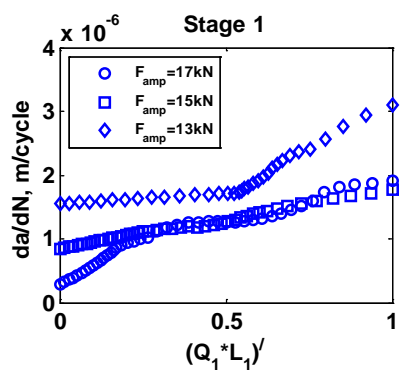

a)

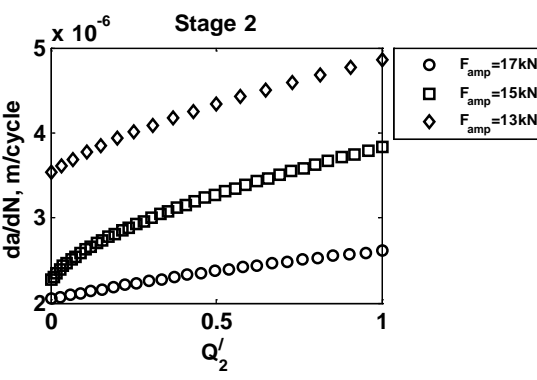

b)

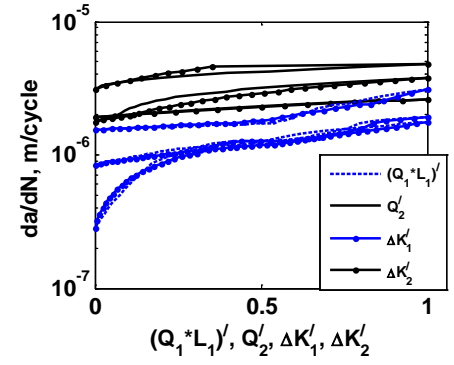

c)

Fig. 9. The dependence between crack growth rate and normalized data of heat flux multiplying by crack length on the first stage of dissipation process (a) and normalized data of heat flux on the second stage of heat dissipation (b); c comparison of normalized data with curve of fatigue crack growth. Data obtained on specimens of dog bone shape with a central hole

Normalization (1) was applied to all experimental data. The goal of this data processing was to find the character of dependence between crack growth rate and heat flux and to compare it with standard Paris law.

Results of data processing are presented in Figures 7-8. Figures 7a, 8a, 9a and Figures 7b, 8b, 9a illustrate corresponding values of $\mathrm{Q} 1 \mathrm{a} 1$ and $\Delta \mathrm{K} 1$ (Stage 1), Q2 and $\Delta \mathrm{K} 2$ (Stage 2) for each types of geometries. Figures 7c, 8c and $9 \mathrm{c}$ demonstrate approximately linear dependence between crack growth rate and normalized values from expression (1).

The results presented in Figures 6-8 exhibit the linear relation for both regimes of crack propagation. The classical Paris regime of crack propagation can be divided into two regimes with different kinetics of energy dissipation. During first regime the crack rate is linear function of crack length and power of heat dissipation $\left(\frac{\partial a_{1}}{\partial N} \sim Q_{1} a_{1}\right)$. The second regime is characterized by generally expected linear relation between crack rate and power of heat dissipation $\left(\frac{\partial a_{2}}{\partial N} \sim Q_{2}\right)$.

\section{Results}

As a result of experimental program it was shown that in the Paris regime the heat dissipation at the crack tip can be divided into two regimes. The first one is characterized by the constant value of heat dissipation form the crack tip. The second regime corresponds to the avalanche-like rise of heat flux. The analysis of experimental data shows:

- under the first regime the crack rate is linear function of crack length and power of heat dissipation $\left(\frac{\partial a_{1}}{\partial N} \sim Q_{1} a_{1}\right)$;

- under the second regime the crack rate is linear function power of heat dissipation $\left(\frac{\partial a_{2}}{\partial N} \sim Q_{2}\right)$.

These results were confirmed by the experiments with different materials and with different specimen geometries.

The physical reason for these results could be the different mechanisms determining the crack propagation at various stress range. The results could allow one to use the value of the heat flux in crack tip area as an alternative way to estimate the crack growth rate independent on loading conditions

\section{Acknowledgements}

This work was supported by the RFBR No. 16-31-00130, 14-01-00122.

\section{REFERENCES}

[1] lino Y., Fatigue crack propagation work coefficient - a material constant giving degree of resistance to fatigue crack growth, Engineering fracture mechanics. - Vol. 12, pp. 279-299, 1979.

[2] Chow CL, Lu TJ., Cyclic J-integral in relation to fatigue crack initiation and propagation, Engineering Fracture Mechanics. - Vol.39, no 1 (1991), pp. 1-20, 1991.

[3] Dowling NE, Begley JA., "Mechanics of crack growth. ASTM STP 590". PA: American Society for Testing and Materials, pp. 83-104, Philadelphia, 1976. 


\subsection{1/qirt.2016.101}

[4] Lindley T. C., McCartney L. N., "Mechanics and mechanisms of fatigue crack growth". Developments in Fracture Mechanics, Applied Science Publishers, London, 1981.

[5] Izumi Y., Fine M. E., Mura T., Energy considerations in fatigue crack propagation, Int. J. Fracture. -Vol. 17, pp.15-25, 1981.

[6] Chakrabarti A. K., An energy-balance approach to the problem of fatigue-crack growth, Engng Fracture Mech. Vol. 10, pp. 469-483, 1978.

[7] Christensen R. M., Wu E. M., A theory of crack growth in viscoelastic materials, Engng Fracture Mech. - Vol. 14, pp. 215-225, 1981.

[8] Bodner S. R., Davidson D. L., Lankford J., A description of fatigue crack growth in terms of plastic work, Engng Fracture Mech. - Vol. 17, pp. 189-191, 1983.

[9] Short J. S., Hoeppner D. W., A Global/local theory of fatigue crack propagation, Engineering Fracture mechanics. - Vol. 33, no 2 (1989), pp. 175-184, 1989.

[10] Memhard D, Brocks W, Frick S., Characterization of ductile tearing resistance by energy dissipa-tion rate, Fatigue Fract. Engng Mater. Sfruct. - Vol. 16, no 10 (1993), pp. 1109-1124, 1993.

[11] Turner CE, Koledni O., Application of energy dissipation rate arguments to stable crack growth, Fatigue Fract. Engng Marer. Struct. - Vol. 17, no 10 (1994), pp. 1109-1127, 1994.

[12] Chudnovsky A., Moet A., Thermodynamics of translational crack layer propagation, Journal of Materials Science. - Vol. 20, pp. 630-635, 1985

[13] Strüwe A., Pippan R., On the energy balance of fatigue crack growth, Computers and Structures. - Vol. 44, no 1/2 (1992), pp.13-17, 1992.

[14] Vshivkov A, Iziumova A, Bär U, Plekhov O., Experimental study of heat dissipation at the crack tip during fatigue crack propagation, Fracture and Stractural Integrity. - Vol. 35, pp.131-137, 2016.

[15] Fedorova A.Yu., Bannikov M.V., Plekhov O.A., Infrared thermography study of the fatigue crack propagation, Fracture and structural integrity.- Vol. 21, pp. 46-53, 2012 